Pedagogía y Saberes No. 51

Universidad Pedagógica Nacional

Facultad de Educación. 2019.pp. 33-48

\title{
Interpretación docente frente a las contribuciones de los estudiantes en el ámbito universitario
}

\section{Artículo de investigación}

\section{Teacher Interpretation against the Contributions of the Students in the University Scope}

Interpretação do professor em face das contribuições do Estudantes no âmbito da universidade

Para citar este artículo:

José Luis Medina Moya* Luz Nelly Rivera Álvarez** Juan Carlos Díaz Álvarez ${ }^{* * *}$

Medina, J. y Rivera, L. y Díaz, J. (2019). Interpretación docente frente a las contribuciones de los estudiantes en el ámbito universitario. Pedagogía y Saberes, 51, 33-48.

* Profesor de la Universitat de Barcelona y doctor en Ciencias de la educación de la misma Universidad e investigador del Grup Formació Docent i Innovació Pedagògica (FoDIP).

Correo electrónico: jlmedina@ub.edu

Código ORCID: orcid.org/0000-0002-9487-9065

** Profesora de la Universidad Nacional de Colombia. Doctora en educación y sociedad de la Universidad de Barcelona e investigadora del Grupo Salud y cuidado de los colectivos.

Correo electrónico: lnriveraa@unal.edu.co

Código oRCID: orcid.org/0000-0001-8750-1155

*** Profesor de la Universidad Nacional de Colombia. Doctor en Bioética de la Universidad El Bosque e investigador del Grupo Salud y cuidado de los colectivos.

Correo electrónico: jcdiaza@unal.edu.co

Código ORCID: httorcid.org/0000-0001-9013-7681 


\section{Resumen}

En este artículo se presentan los resultados de la investigación Saberes disciplinares, saberes pedagógicos y aprendizaje situado: Génesis e influencias mutuas en la enseñanza universitaria, que buscó comprender cómo el profesor interpreta y evalúa las contribuciones del estudiante. Es una investigación cualitativa, microetnográfica, que arrojó las categorías: percepción de la complejidad de las contribuciones, saber experiencial del estudiante, importancia de pedagogías contextualizadas y situadas, la necesidad educativa del estudiante y procesos dialógico-reflexivos en la relación educativa. Se destaca la capacidad docente para diagnosticar la contribución y elaborar una respuesta significativa y contextualizada, en la que implícitamente se conjuga el saber disciplinar, pedagógico y ético.

\section{Palabras clave}

educación en enfermería; formación profesional en salud; acción y raciocinio pedagógico; Conocimiento Didáctico del Contenido (CDC)

\section{Abstract}

This article presents the results of the research Disciplinary knowledge, Pedagogical knowledge and Situated Learning: Genesis and mutual influences in education at the University. The research identified how the teacher receives and evaluates the student's contributions. It is a qualitative research, microethnography, which gave as emerging categories: perception of the complexity of the contributions, the student's experiential knowledge, importance of contextualized and situated pedagogies, the student's educational need, and dialogical-reflexive processes in the educational relationship. It was highlighted the teacher's faculty to diagnose the contribution and also to show significative response within the context produced by the adjustment in the interaction, in which the disciplinary, pedagogical and ethical knowledge combined themselves implicitly.

\section{Keywords}

nursing education; health human resource training; pedagogical reasoning and action; Pedagogical Content Knowledge (PCK)

\section{Resumo}

Neste artigo apresentamos os resultados da pesquisa Conhecimento disciplinar, conhecimento pedagógico e Aprendizagem Situada: Gênese e influências mútuas no ensino na Universidade. A pesquisa identificou como o professor interpreta e avalia as contribuições do aluno. É uma pesquisa qualitativa, micro-etnográfica, que deu como categorias emergentes: percepção da complexidade das contribuições, conhecimento experiencial do aluno, importância das pedagogias contextualizadas e situadas, necessidade educativa do aluno e processos dialógico-reflexivos na relação educativa. Destaca-se a capacidade do professor para diagnosticar a contribuição e elaborar uma resposta significativa e contextualizada, que combina implicitamente conhecimento disciplinar, pedagógico e ético.

\section{Palavras-chave}

educação em enfermagem; formação profissional em saúde; ação e raciocínio pedagógico; Conhecimento Didático do Conteúdo (CDC) 


\section{Marco teórico}

Este artículo presenta resultados del estudio multicéntrico Saberes disciplinares, saberes pedagógicos y aprendizaje situado: Génesis e influencias mutuas en la enseñanza universitaria, en el que participaron 6 universidades de España y Colombia. El estudio analizó las interacciones entre los saberes (pedagógicodisciplinar) del profesor universitario en relación con el discurso del estudiante y con su aprendizaje. Teóricamente el estudio se fundamentó en los planteamientos del Conocimiento Didáctico del Contenido (CDC en adelante), también reconocido por sus siglas en inglés como PCK (Pedagogical Content Knowledge) propuesto por Shulman en 1987 (Acevedo, 2009; Medina y Jarauta, 2013), quien demostró que el CDC es el conocimiento específico que poseen los docentes expertos, y se trata de una combinación adecuada entre el conocimiento de la materia a enseñar y el conocimiento pedagógico y didáctico para promover en el estudiante la comprensión y adquisición de competencias (Acevedo, 2009; Fonseca, 2017; Medina y Jarauta, 2013; Schubert, Carvalho, Costa, Pareto y Souza, 2017). Los estudios del CDC (о PCK) interesan por sus características conceptuales y manifestaciones empíricas. Las características conceptuales han permitido identificar sus elementos constitutivos. Así, Smith y Neale (1989) describen el CDC como un constructo multidimensional con tres componentes: conocimiento del contenido, conocimiento de los errores de los estudiantes en la comprensión de la materia y conocimiento de estrategias de enseñanza. Por su parte, Grossman (2005) incluye en el CDC cuatro dominios: el conocimiento de la comprensión y representaciones que tienen los estudiantes sobre el contenido y sus dificultades de aprendizaje, el conocimiento del currículo, conocimiento de estrategias y de representaciones específicas de los temas por abordar, y conocimiento y creencias sobre los propósitos de la enseñanza de la asignatura. A estos elementos Gess-Newsome y Lederman (1999) añaden el conocimiento del contexto (cultural, curricular y social). No obstante, dada la naturaleza compleja del constructo CDC, se necesitan más estudios empíricos para entender la relación de sus componentes con su construcción en el contexto de la práctica docente (Fonseca, 2017).

Medina y Jarauta (2013) afirman que los docentes expertos muestran gran dominio del contenido, así como la habilidad de buscar en cada momento estrategias adecuadas para su enseñanza en las que se integra y fusiona el saber disciplinar y el saber pedagógico para dar forma y sentido didáctico a lo que enseñan. En su estudio observaron que el profesorado adecuaba el conocimiento científico-disciplinar para que fuera comprensible a sus alumnos (mediante antropomorfismo, metáforas, analogías o ejemplos) y un objetivo fundamental de las explicaciones docentes en el aula era que los estudiantes elaborasen nuevos significados para comprender la materia. En consonancia con Depaepe, Verschaffel y Kelchtermans (2013), lo anterior se explica por la importancia de la exposición oral como opción metodológica mayoritaria en la enseñanza universitaria. Asimismo, Medina y Jarauta (2013) encontraron que un aspecto del CDC poco estudiado en el nivel universitario es el componente dialógico-reflexivo (Yang y Carless, 2013), este componente refleja los procesos dialógicos que se dan en la interacción docente-estudiante, como: la reflexión introspectiva que hace el docente, el diálogo reflexivo cuando comparte su propia reflexión, la reflexión en la acción que es la reorganización de las acciones del docente a partir de su reflexión y la reflexión sobre la acción (Ávila y Medina, 2012; Schön, 1998). Lo anterior justificó el planteamiento y desarrollo de la presente investigación y se correspondió con algunas de las limitaciones que señaló Shulman torno al estudio de PCK, cuando señaló el énfasis en su constructo, con una preocupación más centrada en el razonamiento pedagógico que en la práctica misma (Shulman, 2015, citado por Fonseca, 2017).

Por otra parte, los estudios del CDC (o PCK) han investigado la "dialogicidad interna" o autorreferencial, esto es, la representación (reconstrucción) de ese saber a partir del relato del docente, aún no se ha considerado lo suficiente la importancia de su "dialogicidad externa", esto es, el papel coprotagonista que juegan las contribuciones de los estudiantes en su génesis y despliegue, al igual que sus repercusiones reales en el aprendizaje (Medina y Jarauta, 2013). A partir de ello, se vislumbró la imposibilidad de estudiar el discurso del docente de manera aislada, so pena de fragmentar su naturaleza dinámica y relacional, y la necesidad de su análisis en relación con el discurso del estudiante y su aprendizaje.

El análisis entre las contribuciones discursivas del profesor y las del estudiante fueron objeto del presente estudio, para dar respuestas a las preguntas de investigación en torno a estas interacciones discursivas: ¿Cómo percibe el profesor las contribuciones del estudiante? ¿A qué parte de ellas responde? ¿Cómo es el proceso mediante el que determina a qué prestar atención? ¿Cómo interpreta la contribución del estudiante? Se partió del supuesto de que cualquier interacción discursiva entre profesores y estudiantes se adapta a su contexto, es decir, es situada porque las formas de percibirla, concebirla y ejecutarla se desarrollan de manera conjunta. Se pensó que 
toda interacción profesor-estudiante está en parte mediada por el acoplamiento dinámico (subjetivo e intersubjetivo), momento a momento, de los procesos de percepción, descripción, apreciación, significación y comprensión. Se postuló que el saber pedagógico del docente y el aprendizaje del estudiante tienen un aspecto dinámico en su génesis y contenido. Esa dinámica se mostrará en los resultados. El objetivo planteado fue el describir cómo el profesor percibe, interpreta, evalúa y discrimina lo relevante de las contribuciones del estudiante.

\section{Metodología}

La naturaleza social, simbólica y no lineal del objeto de estudio y los referentes epistemológicos implicaron la adopción de un enfoque onto-epistémico, el interaccionismo simbólico (Blumer, 1969).

Desde esta perspectiva, la naturaleza de los procesos enseñanza-aprendizaje se examinan para su comprensión porque en esos procesos el ambiente se materializa en la fuente de datos del estudio. Metodológicamente el enfoque epistemológico se concretó con el desarrollo de una etnografía de la comunicación (Hymes, 1962), para analizar los eventos comunicativos y factores relevantes lingüísticos, cognitivos y socioculturales relacionados con la interacción comunicativa entre docente y estudiantes. El estudio desarrolló un acercamiento micro etnográfico, basado en la sociolingüística en su vertiente microsocial (Gumperz, 1982). El objetivo de este doble acercamiento fue comprender las interacciones verbales y sociales entre profesores y estudiantes, momento a momento, desentrañando los sentidos $\mathrm{y}$ significados que los participantes ponen en juego en la interacción verbal y no verbal. Tres rasgos del método etnolingüístico lo hacen pertinente para un objeto de estudio que, como el nuestro, es dinámico, relacional y acontece en cortos espacios de tiempo: se realizó una aproximación desde la intersubjetividad como espacio de construcción y organización de los procesos comunicativos y dialógicos. De igual manera, se realizó un acercamiento inductivo ("desde abajo") en su mayoría, que toma como punto de partida el lenguaje en los contextos sociales del aula y su relación con las dinámicas de significación y de aprendizaje. Por último, se ejecutó un acercamiento microanalítico ("puntual") a las formas de construcción y negociación de significados, al igual que a las estrategias de regulación de las interacciones (DiSessa, 2014). Se estudiaron las dinámicas de significación que se sitúan en la experiencia presente e inmediata de profesores y estudiantes (con énfasis en las del docente) y que, cronológicamente, pueden durar segundos o minutos. En esos momentos, desde un punto de vista sociocultural (Vygotsky, 2010) es cuando acontece el aprendizaje en aula, cuando un proceso intersubjetivo se convierte en intrasubjetivo.

Se planteó el análisis secuencial del flujo del discurso, el cual consiste en la construcción de sentidos centrándose en patrones temporales de significación que permite analizar la construcción intra e intersubjetiva de sentido para ir más allá de los límites del análisis semántico del discurso (Nitti, Ciavolino, Salvatore y Gennaro, 2010).

Las fases de estudio fueron:

1. Fase preliminar: Diseño del sistema de comunicación y coordinación del equipo investigador y revisión sistemática de la literatura científica.

2. Fase preparatoria (Selección de participantes): Selección de los participantes que cumplieran los criterios de ser reconocidos como "buen docente" por parte de la comunidad educativa (por resultados de una encuesta dirigida a estudiantes y profesores y con la evaluación de desempeño docente), uso de metodologías participativas y grupos de máximo 50 estudiantes.

3. Fase de trabajo de campo (Procedimientos y técnicas de recogida de información): El conjunto de técnicas de recogida de datos combinó la observación no participante, las notas de campo, la entrevista inmediata de evocación del recuerdo (Fonteyn, Kuipers, y Grobe, 1993) y el método de las transcripciones paralelas (Erickson y Simon, 1993; Wenston y McAlpine, 2002).

a) Entrevistas semiestructuradas biográficoprofesionales (Valles, 1999). Con este tipo de entrevista se obtuvo información sobre fuentes del Conocimiento Didáctico del Contenido, formación disciplinar y pedagógica del docente e información sobre el contexto institucional y curricular.

b) Observación no participante y grabación de las clases (Kawulich, 2006; Valles, 1999). En la observación no participante, afirma Woods (1987), el investigador adopta la técnica de la "mosca en la pared" para observar las cosas tal como suceden, de manera natural, sin la interferencia de su presencia (p. 52). En total se realizaron 8 sesiones de observación, con una duración de 2 a 4 horas. Las observaciones de las interacciones entre el docente y los estudiantes fueron registradas en dos cámaras de vídeo; una enfocada al docente 
y la otra a los estudiantes. Las observaciones estuvieron acompañadas de notas de campo en el aula.

c) Análisis inmediato de la clase y selección de episodios relevantes caracterizados por interacciones discursivas del tipo E-P-E' (estudiante-profesor-estudiante). En las 24 horas posteriores, se realizó un primer análisis del video — el cual contó con un marcador de tiempo que proporcionó referencia cronológica de los episodios relevantes- junto con las notas de campo para identificarlos y profundizar en las entrevistas de evocación del recuerdo.

d) Entrevista de evocación del recuerdo (Charters, 2003; Erickson y Simon, 1993). Esta técnica invita al participante a verbalizar sus acciones mientras las desarrolla (Erickson y Simon, 1993). Dada la imposibilidad de hacer esto durante una clase, al igual que en la mayoría de las investigaciones educativas que han usado esta estrategia (Cotton y Gresty, 2005) se realizó la entrevista dentro de las 48 horas siguientes a la grabación. Las entrevistas se realizaron a cada participante, quienes protagonizaron las interacciones en el aula, la cual consistió en la proyección del video de la clase. A medida que trascurría la proyección, el investigador introducía preguntas orientadas hacia el sentido, significado e intencionalidad de la acción y el discurso de estudiante y profesor (E-P) y sobre las repercusiones en el aprendizaje del estudiante (P-E'). Estas entrevistas a la profesora y a los estudiantes también fueron videograbadas.

e) Transcripciones paralelas (Weston y McAlpine, 2002). Esta técnica permitió documentar cómo los individuos interpretan y responden a una misma experiencia en la que participan; también comprobar la interpretación común y diferenciada que dan a las situaciones estudiadas, lo que enriqueció el análisis de los datos al ser presentados de forma comparativa (Weston y McAlpine, 2002). Para ello, se crearon cinco matrices paralelas bajo los epígrafes tiempo, interacciones de clase, reflexión del docente, reflexión del estudiante y notas de campo del investigador. Se transcribieron de forma sincronizada las interacciones originales y las entrevistas de evocación del recuerdo.

f) Finalización de la recogida de información (saturación). No se tuvo definido un número o tiempo determinado de clases. Al ser el dato centro de atención en la investigación cualitativa, su proceso de recolección finalizó cuando las informaciones recogidas tanto en las grabaciones de clase como en las entrevistas empezaron a ser redundantes (Corbin y Strauss, 2002).

4. Fase analítica. Constituida por:

a) Fase de microanálisis. La unidad de análisis conformada por la interacción E-P-E' incluyó la contribución del estudiante a la que el profesor responde de forma inmediata y cómo esta respuesta es recibida por el estudiante. El enfoque microanalítico exigió altos grados de minuciosidad, se distinguieron en la intervención del profesor (P) tres momentos (identificación-evaluaciónrespuesta) que dieron como resultado la unidad ampliada E- P (i-e-r)-E', tal como se presenta en la tabla 1 :

b) Fase análisis holístico. Tras el microanálisis de los datos, se llevó a cabo un análisis relacional y contextualizado que permitió describir los procesos de pensamiento $\mathrm{y}$ acción pedagógica que contribuyeron de modo efectivo al desarrollo de aprendizajes de alta calidad. Estos resultados fueron triangulados con los profesores participantes y con los especialistas internacionales del equipo de trabajo (Medina, 2014; Taylor y Bogdan, 1987).

\section{Resultados}

El proceso de percepción, interpretación, evaluación y discriminación que realiza el docente delante de las contribuciones de los estudiantes es un proceso dialéctico, dinámico y complejo, tal como se muestra en las categorías emergentes del estudio (Véase tabla 2):

\section{Percepción de la complejidad de las contribuciones de las(os) estudiantes, que requiere poner a disposición el saber disciplinar del docente para responder}

Las contribuciones de los estudiantes, en general, fueron consideradas por las docentes como preguntas o contribuciones complejas, con cierto grado de dificultad para ser respondidas, las cuales exigían poner a disposición el saber disciplinar de la docente para elaborar la respuesta. Las docentes perciben como relevante en la pregunta del estudiante sus hipótesis de comprensión en torno al contenido, 
Tabla 1

Análisis por elementos de la Unidad E- P (i-e-r)-E'

\begin{tabular}{|c|c|c|}
\hline \multicolumn{2}{|c|}{$\begin{array}{l}\text { Unidad de análisis } \\
\qquad \text { E-P(i-e-r)-E' }\end{array}$} & Tipo de análisis y elemento de la unidad analizado \\
\hline Alumno & Contribución & $\begin{array}{l}\text { Análisis del discurso - Elemento E: Identificación inductiva de la tipología } \\
\text { de contribuciones del estudiante a las que el profesor responde. }\end{array}$ \\
\hline \multirow{3}{*}{ Profesor } & Identificación & \multirow{2}{*}{$\begin{array}{l}\text { Análisis del discurso - Elementos E-P(i-e): A partir del relato } \\
\text { introspectivo del profesor sobre su acción, se reconstruyen } \\
\text { los microeventos de percepción, descripción, apreciación, } \\
\text { categorización y significación de la contribución del estudiante. }\end{array}$} \\
\hline & Evaluación & \\
\hline & Respuesta & $\begin{array}{l}\text { Análisis del discurso - Elementos } P(e-r) \text { : A partir del relato introspectivo } \\
\text { del profesor sobre su acción, se reconstruyen los microeventos que } \\
\text { explican el progresivo despliegue de la elaboración de la respuesta. } \\
\text { Análisis del discurso - Elemento } P(r) \text { : Identificación inductiva } \\
\text { de la tipología de respuestas del profesor. }\end{array}$ \\
\hline Alumno & Contribución' & $\begin{array}{l}\text { Análisis del discurso - Elemento } r \text { - E': Identificación inductiva de la } \\
\text { tipología de las reacciones del estudiante a la respuesta del profesor. } \\
\text { Análisis Elementos } E-P(r)-E^{\prime} \text { : Comparación de los estados } \\
\text { cognitivos del estudiante (atencionales, perceptuales, } \\
\text { conceptuales) antes y después de la respuesta del profesor. }\end{array}$ \\
\hline
\end{tabular}

Fuente: elaboración propia.

Tabla 2

Metacategoría y categorías emergentes

\begin{tabular}{|c|c|}
\hline Metacategoría & Categorías emergentes \\
\hline $\begin{array}{c}\text { Percepción e interpretación } \\
\text { docente frente a las contribu- } \\
\text { ciones de los(as) estudiantes }\end{array}$ & $\begin{array}{l}\text { Percepción de la complejidad de las contribuciones de las(os) estudiantes, que } \\
\text { requiere poner a disposición el saber disciplinar de la docente para responder. }\end{array}$ \\
\cline { 2 - 2 } & $\begin{array}{r}\text { Reconocimiento del saber experiencial del estudiante } \\
\text { Percepción de la importancia de contextualizar y situar } \\
\text { al estudiante en la vivencia del paciente }\end{array}$ \\
& Reconocer la necesidad educativa del estudiante \\
\hline Procesos de reflexión en la acción y dialógico-reflexivos \\
en la interacción docente-estudiante
\end{tabular}

Fuente: elaboración propia.

estas favorecen el planteamiento de situaciones dilemáticas y de cuestionamiento, que les permita tomar conciencia de sus necesidades de aprendizaje y de la fragilidad de su conocimiento ante aspectos inusuales y sorprendentes de la materia (Medina y Jarauta, 2013), tal como se evidencia en las siguientes contribuciones de los estudiantes:

“QQué se haría en una situación en donde el paciente no quiere comunicarse con la enfermera?" (Eps 1 Obs 1 P1) ${ }^{1}$

1 Codificación empleada para los verbatim de los(as) participantes: Eps (episodio); Obs (observación); P (profesora); E
“¿Profe, un cambio de actitud puede provocar una reacción asmática?" (Eps 5 Obs 1 P2)

"Profe eso cómo se maneja, digamos si es (pérdida de memoria) de largo plazo o de corto plazo, lo digo porque yo tengo una tía que ya es mayor, y está empezando a tener esos episodios como de demencia, (...) empieza a contar sus historias de cuando joven, pero lo de ahora no lo recuerda." (Eps 9 Obs 2 P2)
(Estudiante); Ent P (entrevista a profesora); Ent Est (Entrevista a Estudiante). 
Ante estas contribuciones las profesoras perciben que elaborar la respuesta resulta difícil, por la complejidad de las preguntas, interpretan que el estudiante tiene un conocimiento previo de la temática, pero se requiere de un saber experiencial y disciplinar para responder:

"Profesora: La contribución fue buena, para mí no era tan fácil responderle porque era algo complejo de explicarle al estudiante y que se necesita de bastante experiencia para poder darle al estudiante una respuesta que fuera satisfactoria. El estudiante es muy puntual, ¿qué hago si no puedo hacerlo porque el paciente tiene tal cosa? (...) Cuando pasa esto ¿qué debo hacer?" (Eps 1 Obs 1 P1 Ent P1)

"Profesora: Yo sabía lo que él quería preguntar, pero elaborar la respuesta un tanto complejo porque si yo no daba una respuesta precisa a lo que él me estaba preguntando, yo lo podía confundir más, y él me estaba era preguntando ¿un cambio de altura puede suscitar una crisis de asma? entonces por eso yo me quedé, como que dije 'Mmm, ¿cómo le respondo a este muchacho, para no confundirlo?'." (Eps 5 Obs 1 P2 Ent P2)

"Profesora: No era una pregunta fácil de contestar. Nuevamente entonces tenía yo que retomar la parte de anatomía y fisiología y decirle 'mire, es que hay unas partes del cerebro, recordemos y retomemos lo que ustedes ya vieron en morfofisiología, y recuerden dónde se fija la memoria'. Entonces ella conectó de una vez eso que estaba viendo en la clase con lo que le pasa a ella en su vida, y creo que eso le ayuda a que quede más claro, para que le pueda brindar cuidados a su tía." (Eps 9 Obs 2 P2 Ent P2)

Las docentes observadas arman un esquema mental en el que ponen a su disposición el saber experto - su conocimiento teórico-conceptual y conocimiento práctico experiencial-y a su vez lo traducen a su saber pedagógico, mediante un proceso de interpretación didáctica y de traducción dialógica. La profesora, dada su experiencia y reflexión sobre la misma, interpreta - de manera didáctica - cuáles son las posibles dificultades que tendría el estudiante, y utiliza esta información para promover la comprensión (Medina y Jarauta, 2013). Presenta su saber experto en un lenguaje comprensible para el estudiante - a través de ejemplos de la vida diaria y uso de anécdotas de experiencias propias-mediante la traducción dialógica y la reelaboración en conceptualizaciones más precisas, por ejemplo, cuando el docente vuelve a explicar el mismo contenido de diversas maneras, utilizando otras palabras para aclarar con mayor profundidad el contenido del tema.

"Tenía que armar todo un esquema en mi cabeza ¿cómo le explico que a veces se suele presentar eso?, en ese esquema yo tengo en cuenta las experiencias que uno ha tenido, manejando ese tipo de pacientes y las causas por las cuales el paciente puede tener ese comportamiento, rápidamente se me viene a mi cabeza el esquema para poderle responder al estudiante, 'mire, usted puede tener un paciente en esta y estas condiciones, pero usted tiene que tratar de intervenirlo de esta manera'. Pues, para que sea un poco más fácil y asequible al entendimiento del estudiante que no tiene una experiencia en un área así, ni una experiencia con ese tipo de pacientes" (Eps 1 Obs 1 P1 Ent P1)

Aquí se evidencia que los docentes ponen en juego su capacidad de focalizar su atención de manera flotante en las ideas del estudiante y en sus propios marcos categoriales, mediante la interpretación didáctica, la traducción dialógica y el uso de historias anecdóticas, manifestaciones propias del Conocimiento Didáctico del Contenido (Medina y Jarauta, 2013).

\section{Reconocimiento del saber experiencial de la estudiante}

Las docentes perciben en las contribuciones de los estudiantes el carácter experiencial que estas contienen. De acuerdo con Díaz (2003), el aprendizaje significativo trasciende la repetición memorística de contenidos inconexos, al buscar construir significado, dar sentido a lo aprendido, y entender su ámbito de aplicación y relevancia en situaciones académicas, reales y cotidianas.

En una profesora se apreció cómo fomentaba el aprendizaje significativo en los estudiantes, al interrelacionar la entidad patológica - el asma, la experiencia del estudiante que vive con esta enfermedad y las características de los cuidadores del paciente con asma- mediante la estrategia pedagógica de partir de la experiencia del estudiante. Esta estrategia le permitió interrelacionar las dimensiones fisiopatológica, psico-emocional y social del asma. De esta manera contribuyó a la comprensión y aprendizaje del tema. A continuación, se presentan dos episodios: 
Tabla 3

Episodios 1 y 2

Descripción de los episodios 1 y 2 videograbados en el aula

E: cuando era niña siempre tuve varias crisis y hace poquito, precisamente cuando entré a la universidad debido al estrés volvió a comenzar. Y comenzó con la bronquitis y empezó otra vez, las crisis las tengo más que todo cuando tengo gripa. Es horrible porque la sensación es que uno no puede respirar y por las noches empiezan a sonar sibilancias. P: ¿Tú la escuchas?

E: Sí y mi hermana que está en el cuarto las escucha también, son fuertes. Esto me lo manejan con salbuta-

mol. Y cuando tengo crisis, con corticoides y con bromuro de ipratropio. Y me hacen terapias respiratorias.

P: ¿Y cómo te sientes tú frente al hecho de tener asma en tu vida?

E: Cuando me dan crisis me siento súper vulnerable, siento que no puedo hacer las cosas que normalmente

hago, sobre todo cuando estoy aquí en la universidad o sea no rindo igual. Y pues el hecho de que, muchas veces

cuando tengo las crisis no puedo salir por la noche, entonces a veces eso influencia un poquito como mi rutina.

P: ¿Y en tu familia como ha sido?

E: Mi mamá es auxiliar de enfermería, entonces ella ha estado muy pendiente de eso, siempre desde chi-

quita me ha cuidado de que no me enferme. También me ha dado muchas vitaminas.

P: ¿Será que si uno toma vitaminas no le da asma? Ahorita veremos ¿hay muchas creencias no?

E: Y también como que intenta fortalecer mi sistema inmunológico. Darme mucha emulsión de Scott (...)

P: ¿No te da miedo que ten den crisis?

E: Sí es que la verdad pues yo... una vez que me dio una crisis tan horrible. ¡En serio! Se me cerró totalmente y yo no podía respirar, me dio cianosis y pues tuvimos que ir a urgencias. (Eps 1 Obs1 P2)

P: ¿Tu mamá es asmática? Escuchen bien lo que sus compañeros están contando, porque ahorita van a correlacionar todo eso, con lo que yo les vengo a contar. Si ustedes quieren aprender de la fisiopatología de una enfermedad, siéntense a hablar con la persona que tiene la enfermedad y ahí van a conectar todo... ¿y que tu mamá es asmática? E: Mi mamá es asmática y a mí me diagnosticaron desde los dos años, vivía hospitalizado bastante seguido por el asma, más o menos mínimo por mes era una semana. La crisis asmática más fuerte que tuve fue como a los 8 o 9 años, de la que tengo recuerdo, comenzó la sibilancia como a eso de las 7 u 8 de la noche, me dijeron 'váyase a acostar, abríguese bien', mi mamá tenía la maña igual como toda buena madre de campo, de que el aceite de tiburón y todo lo que fuera del pescado, sirve para contener los pulmones, entonces ella conseguía aceite de tiburón, una botella que eso destapándolo era horrible. También hacía jugo de naranja con miel y mantequilla. Un poco de remedios caseritos para fortalecer los pulmones. Ese día me dieron todo eso y me levanté como tipo dos o tres de la mañana. Cuando uno tiene asma que es bastante recurrente en ese tipo de situaciones, toca dormir como semisentado.

P: Escuchen eso, él se despertó, el asma lo despertó.

E: Me desperté como a las 3 am, totalmente imposibilitado para respirar, dormía en una habitación con mi otra hermana, y me tocó pegarle a la cama para que ella me viera y ahí sí corra con mis papás al hospital, porque ya me levanté (sonidos y gestos de dificultad respiratoria) ... a lo que pudiera respirar. (Eps 2 Obs 1 P2)

Fuente: datos propios de la investigación.

Aquí la docente hace énfasis y parafrasea fragmentos del discurso del estudiante que considera relevantes para retomarlos, ampliarlos, matizarlos o corregirlos en el desarrollo del tema, por ejemplo: "escuchen eso, el asma lo despertó" “¿Será que si uno toma vitaminas no le da asma?". La docente está atenta al lenguaje verbal y no verbal (actitud y anticipación) de la contribución del estudiante para orientar el diálogo, esto le permite identificar el impacto que ha tenido la enfermedad en su vida y transferir (comunicar) esa realidad al resto de los estudiantes:

"En particular le presté más atención a lo que me expresaba de manera verbal, él me hablaba de los cuidados y muchos de esos cuidados, tal vez no tienen la suficiente evidencia científica. Entonces eso fue importante para mí, para usarlo como elemento en clase para resaltarlo ante los compañeros, los eventos asociados a las hospitalizaciones y a los despertares nocturnos, también fue un elemento relevante." (Eps 2 Obs 1 P2 Ent P2)

"También en alguna parte de la clase hablábamos del rol que tenía el cuidador con el niño, como de la sobreprotección, particularmente en un padecimiento como el asma, él lo hace evidente ahí en su lenguaje no verbal (la docente imita los movimientos de sacudida de las manos del estudiante), lo que deja entrever que la mamá estaba ahí muy pendiente de él y eso lo puede llevar a que se limite, y esto sirve para hablarle a los estudiantes acerca de una de las necesidades que tiene el paciente crónico, que es la limitación." (Eps 2 Obs 1 P2 Ent 2)

"Interpreto que igual su contribución es importante para comprender otras cosas relacionadas con la entidad patológica y con la experiencia de los 
cuidadores, ¿Cómo los cuidadores se preocupan por el paciente? ¿Qué hacen para cuidarlo?” (Eps 2 Obs 1 P2 Ent P2)

La intencionalidad de la estrategia fue acercar al estudiante el contenido abstracto con una experiencia real y concreta, para comprender e interrelacionar la entidad patológica con sus manifestaciones clínicas, con los cuidados requeridos y con las características propias del enfermo y del cuidador; como también, favorecer que el estudiante elabore significados alrededor del cuidado a una persona con asma orientado a las necesidades sentidas de la persona:

"Profesora: El ánimo de la pregunta era que, a partir de la experiencia, ella como que sintiera más cercano eso que yo en abstracto les iba a comentar. Entonces siempre partir en una clase bien sea de la experiencia de los estudiantes o mostrándoles una historia real de una persona que viva con la condición de cronicidad, hace que ellos puedan entender una entidad patológica, pero más que eso, es situarse en ese escenario futuro de cuidar a una persona en esa condición y entender que la persona, aunque la enfermedad es muy importante, la persona necesita un cuidado de enfermería que esté enfocado a sus necesidades sentidas." (Eps 1 Obs 1 P2 Ent P2)

Partir de la experiencia de vida del estudiante permite fijar y correlacionar los conocimientos con el cuidado de la persona asmática. Además, se denota cómo la contribución del estudiante aporta a la comprensión de la entidad patológica, a los cuidados e implicaciones de los cuidadores y al aprendizaje contextualizado:

"Utilizar esa estrategia de preguntarle a los estudiantes si alguno ha padecido o alguien en su familia ha padecido la enfermedad o si conoce a alguien, esto les permite fijar un poco más los conocimientos relacionados con el cuidado de la persona con esa condición. Y les hago algunas preguntas orientadoras ¿Cuál es el tratamiento? ¿Qué síntomas había tenido? ¿Qué sentía ella?" (Eps 2 Obs 1 P2 Ent P2)

"Profesora: Interpreto que la contribución que ella hace a la clase es sumamente importante. Porque no tendría sentido que yo les cuente una cantidad de cosas, digamos como desconectadas o como fuera de contexto. Pero cuando yo sitúo la situación de una persona con asma. Y ese contexto ya tiene, en este caso un nombre, ya tiene una experiencia una vivencia. La contribución que hace la estudiante es muy importante para que los compañeros se puedan conectar con el tema." (Eps 1 Obs 1 P2 Ent P2)
La intencionalidad pedagógica de partir de la experiencia del estudiante trasciende la necesidad de conocimiento del estudiante y del grupo buscando particularizar, personalizar y acoger la experiencia mediante la empatía didáctica:

"Profesora: Yo he tenido la experiencia de tener a Juan en una clase. Cuando yo entré a la clase yo ya sabía que él tenía asma, siento yo que él necesita expresarlo. También se lo pregunté en clase, por la necesidad colectiva de los compañeros de saber acerca del tema de lo que les iba a hablar ese día en particular, pero yo también tenía la intencionalidad en ese momento de particularizarlo a él y que él sepa que yo como docente sé cuál es su condición y que estoy ahí por si él necesita algo." (Eps 2 Obs 1 P2 Ent P2)

Esta empatía didáctica permite a la profesora conocer y conectarse con la experiencia del estudiante y asumir la presencia y el estar-ahí como un rasgo importante de su pedagogía. Le asigna importancia al vínculo físico, a la mirada, la escucha y a la atención pedagógica: "Creo que el vínculo físico es importante, estar cerca, mantener la conexión visual" (Eps 2 Obs 1 P2 Ent P2). Esta conexión visual trasciende hacia mirar quién es, qué necesita el estudiante y entablar una conexión emocional con el grupo de estudiantes.

Parafraseando a Díaz (2003), un supuesto instruccional motivacional para fortalecer en los estudiantes de enfermería su razonamiento clínico alrededor del cuidado de la persona crónica, en escenarios auténticos (de la vida real), se mejora considerablemente a través de dos dimensiones: 1) Dimensión Relevancia cultural: Una enseñanza que emplee ejemplos, ilustraciones, analogías, discusiones y demostraciones que sean relevantes para las culturas a las que pertenecen o esperan pertenecer los estudiantes. En las docentes observadas encontramos un análisis colaborativo de situaciones clínicas relevantes y reales. 2) Dimensión Actividad social: Una participación tutorizada en un contexto social y colaborativo de solución de problemas, con ayuda de mediadores como la discusión en clase, el debate, el juego de roles y el descubrimiento guiado.

Wassermann (1994) define los casos como:

Instrumentos educativos complejos que aparecen en forma de narrativas. Un caso incluye información y datos (psicológicos, sociológicos, científicos, antropológicos, históricos, observacionales) así como material técnico. Los buenos casos se construyen en torno a problemas o "grandes ideas", es decir, aspectos significativos de una materia o asunto que 
garantizan un examen serio y a profundidad. Las narrativas se estructuran usualmente a partir de problemas y personas de la vida real. (Wassermann, 1994, citado por Díaz, 2003, p. 9).

Además, los casos "promueven las habilidades de aplicación e integración del conocimiento, juicio crítico, toma de decisiones y solución de problemas en los alumnos" (Díaz, 2003, p. 9).

La enseñanza de las docentes participantes se caracteriza por promover en el estudiante empoderamiento (facultamiento) para intervenir de modo activo en su aprendizaje, pensamiento reflexivo y crítico, responsabilidad en el acto de cuidar a otra persona, aprendizajes significativos, y además, la generación de un ambiente de empatía, confianza y motivación en el grupo.

\section{Percepción de la importancia de contextualizar y situar al estudiante en la vivencia del paciente}

Como se ha mencionado, la enseñanza de las docentes se caracteriza por emplear pedagogías de contextualización (Benner, Sutphen, Leonard y Day, 2010), buscan situar al estudiante en la vivencia del paciente, a través del uso de ejemplos y anécdotas provenientes de su experiencia, dejando ver que ellas reconocen la importancia de entregar una respuesta clara y situada, para generar un aprendizaje situado (Lave y Wenger, 1991). Para Díaz (2003, p. 2) "la cognición situada parte de la premisa de que el conocimiento es situado, es parte y producto de la actividad, el contexto y la cultura en que se desarrolla y utiliza". Por tanto:

la enseñanza situada, destaca la importancia de la actividad y el contexto para el aprendizaje y reconoce que el aprendizaje es, ante todo, un proceso de enculturación en el cual los estudiantes se integran gradualmente a una comunidad o cultura de prácticas sociales. En esta misma dirección, se comparte la idea de que aprender y hacer son acciones inseparables. (Díaz, 2003, p. 2).

Tal como se muestra a continuación:

"Hay muchas estrategias que desde la experiencia uno ha utilizado, por eso tocaba como ambientar un poco para que se situara él sobre qué estaba viviendo el paciente, o sea llevarlo a cosas muy de la vida diaria, para contextualizarlo o situarlo ahí en lo que de pronto el paciente estaba viviendo para poderle responder." (Eps 1 Obs 1 P1 Ent P1)

La docente integra el conocimiento teórico-conceptual con el conocimiento práctico-experiencial y la intención pedagógica (y ética) de que ese conocimiento sea significativo para el estudiante:
"Tenía que armar todo un esquema en mi cabeza ¿cómo le explico que a veces se suele presentar eso?, en ese esquema yo tengo en cuenta las experiencias que uno ha tenido, manejando ese tipo de pacientes y las causas por las cuales el paciente puede tener ese comportamiento, rápidamente se me viene a mi cabeza el esquema para poderle responder al estudiante, 'mire, usted puede tener un paciente en esta y estas condiciones, pero usted tiene que tratar de intervenirlo de esta manera'. Pues para que sea un poco más fácil y asequible al entendimiento del estudiante que no tiene una experiencia en un área así, ni una experiencia con ese tipo de pacientes." (Eps 1 Obs 1 P1 Ent P1)

En este orden de ideas, desde una visión situada, se aboga por una enseñanza centrada en prácticas educativas auténticas, las cuales requieren ser coherentes, significativas y propositivas; en otras palabras: "simplemente definidas como las prácticas ordinarias de la cultura" (Brown, Collins y Duguid, 1989, p. 34, citados por Díaz, 2003, p. 3).

"Acuérdense que hay pacientes que se tornan, por la misma falta de sueño, ruido en la unidad, que lo estén despertando tres o cuatro veces, que no lo dejen dormir, todo eso genera en el paciente ¿qué?, respuestas de angustia y ansiedad, que pueden ser agresivas frente a uno, 'ya no quiero que me hagan nada, ya no quiero'. Muchas veces los pacientes son muy agresivos, pero uno con quedarse al lado de ellos un rato en silencio, puede ir bajando esa ansiedad. Muchas veces ellos le están pidiendo a uno a gritos que los apoyen, que los ayuden y responden es agresivamente, pero uno con un silencio va entrando poco a poco al paciente y le va llegando al paciente, y a veces uno se queda callado, ahí quietico y al rato le contesta el paciente 'que quiere hablar'. Pero toda esa situación que él está viviendo lo hace responder de esa manera, hasta decir 'no quiero ya nada."' (Eps 1 Obs 1 P1)

La pretensión de la docente es la demostración y el decir (Schön, 1992, 1998). El estudiante capta lo que la profesora quiso dar a entender, interpreta la complejidad del silencio y de la presencia para abordar a una persona que no acepta el cuidado de enfermería. La respuesta de la docente lleva al estudiante a hacer una transferencia del conocimiento al ámbito clínico y a dimensionar aspectos psicoemocionales del cuidado de enfermería, además, modifica su visión mecanicista a una integrativa del cuidado, ya no lo percibe como un cuerpo, un objeto, sino como un sujeto de cuidado, tal como lo denota el estudiante en su afirmación: "Ahí es donde yo le comienzo a dar importancia, porque una cosa es ir a 
fijarse en un cuerpo y otra cosa es fijarse en el cuerpo y su entorno, en las partes psicosocial y emocional." (Eps 1 Obs 1 P1 Ent Est)

\section{Reconocer la necesidad educativa del estudiante}

En este apartado se presenta cómo las docentes discriminan en las contribuciones del estudiante lo que van a considerar relevante para elaborar su respuesta. El proceso de discriminación supone tomar una decisión, el límite de lo relevante e irrelevante. El análisis in situ de la contribución del estudiante se da en términos disciplinares. Por ejemplo, las docentes discriminan como relevante de las contribuciones aquellos aspectos que revelan sus necesidades educativas, a través de las preguntas o contribuciones que los estudiantes realizan al mostrar sus hipótesis de comprensión (Medina y Jarauta, 2013) o situaciones que les resultan problemáticas.

A continuación, se presenta cómo la docente lanza preguntas a los estudiantes sobre el monitoreo invasivo e identifica hipótesis de comprensión inadecuadas de los estudiantes

Tabla 4

Episodios 3

Descripción del episodio 3 videograbado en el aula

"P: ¿Y desde lo invasivo qué tenemos?

E1: Los gases arteriales.

P: Los gases arteriales, la PVC, ¿qué más?

E2: Líneas arteriales.

E3: Las tomografías, el tac se hacen

con contraste y todo eso.

P: No, eso sería más medios diagnósticos, más

que sistemas de monitoreo, eh ¿qué más?

E4: ¿Swan Gans?

P: Uso de catéter de Swan Gans, o sistemas de

monitoreo invasivo para medir el gasto cardiaco.

E3: ¿Entubar?

$\mathrm{P}:$ ¿para monitorear?..

E3 (risas): con el capnógrafo se pone (ríe profesora)"

(Eps 9 Obs 2 P1 Ent P1)

Fuente: datos propios de la investigación.

La interpretación de la profesora respecto de la contribución del estudiante señala que este presenta varias hipótesis inapropiadas del contenido:

"Cuando el estudiante dice que un medio de diagnóstico como un sistema de monitoreo, dije 'no, aquí hay que aclarar, no sea que piensen que todo lo que le hacen al paciente son sistemas de monitoreo', luego cuando dijo la intubación, entonces viene otra confusión diferente que es sistemas terapéuticos, entonces poderle aclarar al estudiante que eso pertenece a otro grupo de cosas." (Eps 9 Obs 2 P1 Ent P1)

Este episodio evidencia la sintonización estructural, uno de los rasgos del CDC, que refleja ese carácter recíproco que adquiere la interacción pedagógica entre docente y estudiante. La docente tiene en cuenta que el estudiante está explorando, tiene confusión, muestra sus hipótesis de comprensión, por tanto, su respuesta es exploratoria. La profesora capta —esa cuestión prosódica, pragmática-. Por tanto, se revela esa sintonización estructural entre la interacción dialógica docente-estudiante, enmarcada en un ambiente pedagógico de confianza, respeto y aceptación:

"El estudiante me está dando algunas opciones que no eran reales, no aplicaban para lo que yo estaba preguntando, pero lo hacía de una forma tranquila, espontánea, entonces de esa misma forma pues yo lo retomé y le di a entender que no era eso, porque estaba refiriéndome era a otra cosa." (Eps 9 Obs 2 P1 Ent P1)

Cabe diferenciar que la sintonización puede ser del estudiante o de la profesora. En el primer caso, se representa cuando el estudiante expone sus hipótesis de comprensión, complementa y pregunta. En el segundo caso, cuando la profesora parte de las hipótesis de comprensión del estudiante, las diagnostica y decodifica para elaborar su respuesta, en un trabajo activo que facilita la comprensión. A su vez, en su respuesta hay una mezcla entre ciencia y ética, que se aprecia cuando expresa "afortunadamente surgen esas ideas": "Me sorprendió, porque yo pensé que los estudiantes tenían claro un concepto que era básico, pero afortunadamente surgen estas ideas para poder aclarar un poco más." (Eps 9 Obs 2 P1 Ent P1)

Lo relevante es esa confusión del concepto, la profesora adopta una actitud ética del discurso porque elabora nuevos significados y aclara la comprensión inadecuada del estudiante bajo la perspectiva de la responsabilidad y su dignificación al acoger sus hipótesis de comprensión. Por lo tanto, no se puede separar la dimensión teórica-conceptual de la moral y emocional. Este es un ejemplo que muestra un buen diagnóstico de la profesora, teñido de ese aspecto moral en el que no se evidencia un juicio normativo hacia el estudiante:

"Cuando el estudiante le da a uno una respuesta errada, uno no puede realizar un juzgamiento público porque eso haría que el estudiante nunca más participe y de pronto no aclare sus dudas, lo que yo generalmente hago es retomar del error del estudiante, clarificar y si él dijo algo de manera muy positiva 
realzarlo para que el estudiante no vaya a quedar como: 'oiga, no puedo abrir la boca, cada vez que diga algo el profesor me va a juzgar'." (Eps 9 Obs 2 P1 Ent P1)

Esto en términos de aprendizaje del estudiante tiene repercusión:

"Estudiante: Ella lo hizo de tal manera que me ayudaba más a mi autoestima que a la pregunta en sí. Para que seas un buen enfermero o trabajar en lo que quieras, necesitas tener una buena autoestima, que te apoye la gente que está alrededor. Y yo creo que podría habernos dicho: '¡no!, eso no tiene nada que ver', y ya solo era la autoestima de uno mismo, el saber contestar a la gente y tener empatía, más que la respuesta sea correcta y clarificar." (Eps 9 Obs 2 P1 Ent Est)

Otro episodio que destaca el papel de las hipótesis inadecuadas de comprensión del estudiante como marcador de reconocimiento (Balslev, Vanhulle y Tominska, 2011) relevante para la profesora, se presenta a continuación:

Tabla 5

Episodios 4

Descripción del episodio 4 videograbado en el aula

“P: Juan... ¿puedes contarnos tu experiencia de atopia? ¿Tu historia personal de atopia? (Estudiante no comprende la pregunta y hace gesto a la docente; la docente le muestra su mano y hace un movimiento circular detallando que le está preguntando por una alteración en la piel).

E: A razón del asma tengo las... (estudiante hace gestos con la lengua que denotan confusión) inmunoglobulinas están un poco más bajas, entonces tengo la facilidad de generar dermatitis, entonces por lo que las mismas defensas se encuentran mucho más deterioradas, los daños se ven muy fuertes, entonces el daño es mucho mayor en la piel." (Eps 4 Obs 1 P2)

Fuente: datos propios de la investigación.

En este episodio, la docente discrimina como marcadores de reconocimiento de la contribución el papel de las inmunoglobulinas (Ig) en la atopia y el asma. Además, revela que el estudiante presenta confusión respecto al aumento o disminución de las Inmunoglobulinas. Estas hipótesis de comprensión inadecuadas son una oportunidad de aprendizaje para fijar la atención del estudiante y del grupo, lo que algunos autores han denominado la pedagogía del error (De la Torre, 2004; Guerrero, Castillo, Chamorro y Gil, 2013).
"Lo que pensé en ese momento fue que Juan tiene desconocimiento de los términos científicos con los que se denomina a las condiciones que se asocian con un niño asmático, los factores que aumentan el riesgo de que el niño tenga asma. Y pues él lo deja ver ahí, cuando yo le pregunté particularmente por la atopia, él no sabía qué le estaba preguntando. Pero en este momento pienso que incluso es bueno para la clase, la equivocación. Es una pausa dentro de la clase que les permite fijar la atención y entender que esa palabra atopia tiene que ver con una manifestación clínica en la piel." (Eps 3 Obs 1 P2 Ent P2)

"Me sorprendió que ellos no le tengan miedo a equivocarse dentro del aula de clase, porque a veces en nuestra cultura somos como prevenidos como a no mostrar nuestra vulnerabilidad, como a mostrar que sabemos." (Eps 3 Obs 1 P2 Ent P2)

La docente presta atención a cómo participa el estudiante en el aula, a esa actitud tranquila, segura y que no tiene miedo a equivocarse y mostrar su vulnerabilidad delante de la profesora y el grupo, a lo que el estudiante informa como, por ejemplo, al desconocimiento respecto al concepto de atopia y el papel de las Ig en esta patología.

"El habló de que sus defensas estaban bajas, entonces yo retomé el tema de las defensas. Eso fue relevante hablar de qué eran las defensas y qué era la parte inmunológica. (...) Identificar también que él lo asociaba como un factor predisponente del asma, como que su atopia fue lo que le causó el asma o viceversa. $\mathrm{Y}$ de pronto retomar y decir 'no es que una cosa causa la otra, sino que se asocian'." (Eps 3 Obs 1 P2 Ent P2)

Por otro lado, presta atención al lenguaje no verbal del estudiante, al uso del lenguaje coloquial al referirse a su experiencia de vida y experiencia cultural. El uso de términos coloquiales o populares se constituye en un elemento de gran importancia para brindar educación para la salud a la comunidad y esta confrontación de términos genera otros aprendizajes:

"Me llama la atención que cuando les hago preguntas relacionadas con la experiencia, ellos utilizan un lenguaje coloquial. No hablan de los linfocitos, no hablan de los glóbulos blancos. Ellos empiezan a utilizar otros términos más del común 'que tenía las defensas bajas'. Que finalmente, también es importante porque ellos tendrán que comunicarse con otras personas en esos términos. Y pues es tan importante que manejen el término científico como el término popular, que utiliza la población en general para referirse a ese tipo de cosas." (Eps 3 Obs 1 P2 Ent P2) 


\section{Procesos de reflexión en la acción y dialógico-reflexivos en la interacción docente-estudiante}

Otro aspecto que señalan las docentes como relevante en la contribución de los estudiantes, es su capacidad reflexiva y de análisis al lograr interrelacionar los conceptos teóricos con un ejemplo o anécdota del cuidado enfermero. En el siguiente episodio se observa cómo la intencionalidad comunicativa del estudiante es informar que el juicio clínico es la capacidad de anticipación que el profesional debe tener para evitar complicaciones en la evolución clínica del paciente:

"Uno que tiene que tener, como un ojo clínico como anticiparse a los hechos, para que el paciente más adelante no vaya a sufrir de pronto complicaciones (...) digamos un paciente está taquicárdico, taquipneico, pero no ha hecho fiebre, muy seguramente después va a hacer fiebre y puede ser un signo de que está haciendo alguna infección o alguna cosa similar." (Eps 7 Obs 2 P1 Ent Est).

El proceso de discriminación e interpretación de la profesora contiene los siguientes elementos: presta atención a la participación del estudiante (el qué y cómo participa); destaca su capacidad para extrapolar el concepto "juicio clínico" en un ejemplo diario; discrimina como relevante la reflexión y análisis del estudiante al poner un ejemplo; presta atención al lenguaje corporal en sus movimientos de afirmación; y por último, retoma la contribución del estudiante para complementar y explicar de manera dinámica y en una especie de bucle reflexivo el concepto teórico y el ejemplo práctico, es decir, se observa en la enseñanza un movimiento de lo simple a lo complejo. Se podría afirmar que este episodio evidencia una sincronía educativa entre docente y estudiante.

"Lo que está diciendo su compañero es muy importante, el juicio clínico tiene que ver con esa reflexión, con ese análisis, esa interpretación, esa relación que yo pueda hacer de los datos que estoy obteniendo, para emitir un juicio y decir 'este paciente probablemente tiene un riesgo de infección, o probablemente este paciente está pasando por un estado de ansiedad, o este paciente tiene un riesgo de hacer un gasto cardiaco disminuido'. Yo empiezo a correlacionar todo, a interpretar, a reflexionar y emito un juicio cuando genero un diagnóstico o identifico un problema de un paciente." (Eps 7 Obs 2 P1).

El uso de preguntas abiertas acompañada de una situación clínica problematiza el conocimiento y expone la comprensión del estudiante, genera en él un pensamiento reflexivo e integrador de conceptos previos que lo conecta a la situación actual, para promover un aprendizaje situado y contextualizado: "Al ponerles esa pregunta abierta, el estudiante tuvo la capacidad de análisis, o sea que sí estaba comprendiendo lo que se estaba viendo." (Eps 11 Obs 3 P1 Ent P1).

Otro ejemplo del uso de preguntas que tienen como objetivo ser una sacudida reflexiva (van Zee y Minstrell, 1997b) para elicitar el pensamiento del estudiante, se aprecia en el siguiente episodio:

Tabla 6

Episodios 5

Descripción del episodio 5 videograbado en el aula

"P: ¿quién sabe cuál es la diferencia entre delirio y delirium?

E1: El delirium es un proceso, por así decirlo, que tiene que ver más con una causa orgánica. Mientras que el delirio es todo lo contrario. Mientras que el delirio tiene más que todo raíces (E3: neurológicas), no tanto de neuro sino mentales. Entonces digamos que en urgencias nos encontramos con dos pacientes. Los dos pacientes presentan un cuadro sintomatológico parecido, pero al momento de hacer el descarte nos damos cuenta, que bien uno puede tener una fiebre que puede estar ocasionando que presente alucinaciones o presente paranoias. Mientras que en la otra persona hemos descartado todas las causas orgánicas, hemos hecho de hacer exámenes de sangre y no hemos dado como una causa, entonces suponemos que sí es un delirio. P: Bueno, Daniel lo está pensando desde los factores que desencadenan el delirio o el delirium. ¿Liliana?

E2: Es que el delirium es algo que se da

de forma aguda. (Profesora: ¿cuál?)

E2: El delirium, porque pues lo que decía Daniel, puede ser desencadenado por algún traumatismo, puede ser desencadenado por un síndrome de abstinencia. Pues me han enseñado que la fiebre está como en prueba de si es o no es el delirium. Pero es como una de las diferencias grandes. El delirium es un proceso agudo, ya cuando la persona se le ha resuelto su causa orgánica ya deja de tener toda la sintomatología del delirium. En cambio, el delirio ya es un proceso mental, que tiene un proceso más largo, que tiene sus épocas de crisis que está bien." (Eps 6 Obs 2 P2)

Fuente: datos propios de la investigación.

La profesora identifica en la contribución del primer estudiante, que su respuesta está organizada en torno a los factores desencadenantes del delirio y el delirium. Como experta, detecta que el estudiante titubea respecto a las causas del delirio y delirium, e identifica que comprende la diferencia de estas dos entidades, pero presenta cierta dificultad para expresarlo en palabras. Usa la estrategia de pedirle un ejemplo para que tenga la responsabilidad de pensar de nuevo y obtener una mayor elaboración de su pensamiento (van Zee y Minstrell, 1997b), en 
lugar de evaluar la respuesta del alumno y pasar al siguiente punto en una agenda fija. La profesora "capta" el significado del enunciado del alumno y "arroja" la responsabilidad de pensar de nuevo, no solo a los estudiantes que hablan, sino también a todos los alumnos de la clase (van Zee y Minstrell, 1997b): "Siento que él tenía claro la diferencia entre delirio y delirium, cuando intentó explicarlo a los compañeros. Por eso también le pedí que diera un ejemplo para que los compañeros pudieran afianzar un poquito más el concepto." (Eps 6 Obs $2 \mathrm{P} 2$ Ent P2).

La profesora permite al estudiante concretar el conocimiento abstracto con el que partió en un ejemplo práctico. Esto es lo que van Zee y Minstrell (1997b, p. 228) denominan "seguir la dirección del pensamiento del estudiante". El saber experto docente le permite identificar en el estudiante su intencionalidad comunicativa, se da cuenta por dónde va el planteamiento del estudiante, lo sintetiza - concreta - y le asigna un significado (Santibáñez, 2015), característica importante para que se dé un acoplamiento estructural en el proceso educativo. Estos elementos dialógico-reflexivos contribuyen a la comprensión y organización temática de los participantes:

"Bueno, Daniel lo está pensando desde los factores que desencadenan el delirio o el delirium" (Eps 6 Obs 2 P2 Ent P2).

$\mathrm{Al}$ asignar este significado a la contribución del estudiante, lo estimula para que identifique otro sentido, esta estrategia de repetir el comentario del estudiante es esencial, porque proporciona la oportunidad de fomentar su pensamiento a través de comentarios reflexivos - tipo sacudida reflexiva (van Zee y Minstrell, 1997b) - y la negociación de significados.

En esta negociación participa también la segunda estudiante, para complementar la diferencia de estas dos entidades a partir de discriminar el tiempo de presentación patológica: "agudo o crónico" (agudo para la entidad del delirium y crónico para la entidad del delirio).

Es un claro ejemplo de la sincronización estructural de la comunicación entre docente y estudiante. Para van Zee y Minstrell (1997a; 1997b) estas conversaciones e interacciones son definidas como negociación de significados y sentido cuyas funciones son: aclarar sus significados, considerar una variedad de puntos de vista de manera neutral y monitorear la discusión.

El episodio muestra esa negociación de significados y la construcción conjunta de saberes. Se observan en forma clara los procesos de deliberación, mediación y ayuda ajustada a las necesidades del alumno y el grupo, en el que se promueve el aprendizaje colaborativo. Tal como afirma Díaz (2003):
En un modelo de enseñanza situada, resaltarán la importancia de la influencia de los agentes educativos, que se traducen en prácticas pedagógicas deliberadas, en mecanismos de mediación y ayuda ajustada a las necesidades del alumno y del contexto, así como de las estrategias que promuevan un aprendizaje colaborativo o recíproco. (p. 4).

“Después la profesora pidió preguntas a otros estudiantes para que pudiésemos construir mejor el concepto. Luego fue la intervención de una de mis compañeras, si mal no recuerdo Lilian. Ella me ayudó y fue cuando ya hubo, por así decirlo, una mayor congruencia entre las ideas que yo quería comunicar." (Eps 6 Obs 2 P2 Ent Est 1).

“Me gustó como ella lo planteó primero, para ver qué conocimientos teníamos nosotros y con base a ello trabajar." (Eps 6 Obs 2 P2 Ent Est 2).

"Si bien (la profesora) condujo hacia una respuesta, creo que fue más el constructo que hicimos entre nosotros (los estudiantes). Para haber llegado a esa conclusión. No fue tanto de una parte implícita por la profesora sino más que todo nuestra. (...) Y las observaciones que dio la profesora fueron pertinentes ya que fue concreta y nos ayudó a todos a entrar en la misma sintonía, y en que no hubiese dificultad al entender el tema." (Eps 6 Obs 2 P2 Ent Est 1).

“Considero que, por parte del estudiante, para que se brinde esa discusión, es necesario que nosotros (estudiantes) nos preparemos." (Eps 6 Obs 2 P2 Ent Est 1).

\section{Conclusiones}

Las siguientes conclusiones tienen diversas acepciones puesto que el proceso presenta múltiples variantes a la luz de la teoría contemplada en el CDC o PCK, estas se resumen a continuación:

En el proceso de enseñanza aprendizaje, el estudiante se encuentra en una fase exploratoria y muestra permanentemente las hipótesis de comprensión que elabora alrededor del contenido. Estas hipótesis muestran a las docentes la forma como el alumno está comprendiendo y son la "materia prima" con la cual trabajan, para fijar la atención de los estudiantes, para remarcar, corregir y/o profundizar aspectos esenciales en el aprendizaje significativo. Por tanto, resulta esencial elicitar el pensamiento del estudiante mediante la pregunta problematizadora, la solución de problemas y el conflicto cognitivo con ayuda de mediadores como la discusión en clase, el debate, el juego de roles y el descubrimiento guiado.

La capacidad del docente para diagnosticar la contribución del estudiante, elaborar una respuesta significativa y contextualizada delante de esta contribución 
y lograr un aprendizaje significativo muestra una especie de acoplamiento estructural en la interacción dialógica entre docente-estudiante y esta, a su vez, es un rasgo del Conocimiento Didáctico del Contenido (CDC). En esta sintonización estructural, la docente pone en juego su conocimiento teórico-conceptual, conocimiento práctico experiencial, es decir, su saber disciplinar. Por tanto, en su hacer educativo se integra su saber disciplinar, con su saber pedagógico y una preocupación ética por acoger y respetar los procesos vitales de aprendizaje del estudiante.

Las estrategias pedagógicas empleadas por las docentes buscaron reconocer el saber experiencial del estudiante, con la intencionalidad pedagógica de presentarle un conocimiento contextualizado, significativo e interconectado con la práctica enfermera. Las docentes se presentaron como postuladoras de problemas (reales y significativos) que permitieron generar cuestionamientos relevantes, así como negociación de significados y de sentido que favorecieron al estudiante para aclarar sus significados, considerar una variedad de puntos de vista de manera neutral y tener un mapa que les permita tomar decisiones y desenvolverse delante de las situaciones de la práctica.

Las pedagogías que asumen un carácter contextualizado y situado promueven en el estudiante empoderamiento para participar de manera activa en su proceso de aprendizaje, fomentan el pensamiento reflexivo y crítico, la toma de conciencia respecto a la responsabilidad y la competencia profesional requerida en el acto de cuidar a otra persona. Se trata de una enseñanza centrada en experiencias auténticas, situadas y motivantes, que promueve un aprendizaje significativo, participativo y colaborativo.

Por último, generar en el aula un ambiente de empatía, confianza y motivación resulta fundamental para el aprendizaje significativo. Permite al docente reconocer y conectar con la experiencia del estudiante; acompañar y acogerle delante de sus hipótesis de comprensión desde una ética del respeto y la preservación de la dignidad del Otro(a); particularizar y personalizar la enseñanza reconociendo la potencialidad y/o necesidad propia de cada estudiante; asignar la debida importancia a aspectos como la presencia, el vínculo físico, la mirada, la escucha y la atención pedagógica.

\section{Referencias}

Acevedo, J. (2009). Conocimiento didáctico del contenido para la enseñanza de la ciencia (I): El marco Teórico. Revista Eureka sobre enseñanza y divulgación de las Ciencias, 6 (2), 164-189. Recuperado de https://revistas.uca.es/index.php/eureka/article/view/3698/3286
Ávila, N. y Medina, L. (2012). El Análisis Dialógico del Discurso (ADD) y la teoría de la enunciación: descubriendo la tensión dialógica en los discursos de profesores secundarios chilenos. Estudios de Psicología, 33 (2), 231-247. doi:10.1174/021093912800676466

Balslev, C., Vanhulle, S. y Tominska, E. (2011). A discursive approach to recognition in the practicum. McGill Journal of Education, 46 (1), 23-39.

Benner, P., Sutphen, M., Leonard, V., y Day, L. (2010). Educating nurses. A call for radical transformation [1a. ed.]. United States of America: Jossey-Bass.

Blumer, H. (1969). Symbolic Interactionism. Englewood Cliffs: Prentice-Hall.

Charters, E. (2003). The use of think-aloud methods in qualitative research: An introduction to think-aloud methods. Brock Education, 12 (2), 68-82.

Corbin, J. y Strauss, A. (2002). Bases de la investigación cualitativa: Técnicas y procedimientos para desarrollar la teoría fundamentada. Antioquia, Colombia: Universidad de Antioquia.

Cotton, D. y Gresty, K. (2005). Reflecting on the think-aloud method for evaluating e-learning. British Journal of Educational Technology, 37 (1), 45-54.

De la Torre, S. (2004). Aprender de los errores. Buenos Aires, Argentina: Magisterio del Río de la Plata.

Depaepe, F., Verschaffel, L. y Kelchtermans, G. (2013). Pedagogical content knowledge: A systematic review of the way in which the concept has pervaded mathematics educational research. Teaching and Teacher Education, 34, 12-25.

Díaz, F. (2003). Cognición situada y estrategias para el aprendizaje significativo. Revista Electrónica de Investigación Educativa, 5 (2). Recuperado de http:// redie.ens.uabc.mx/vol5no2/contenido-arceo.html

DiSessa, A. A. (2014). The construction of causal schemes: Learning mechanisms at the knowledge level. Cognitive Science, 38, 795-850.

Erickson, K. y Simon, H. (1993). Protocol analysis: Verbal reports as data. Boston: MIT Press.

Fonseca, G. (2017). El conocimiento pedagógico del contenido en profesores de biología: Una revisión documental. Biografía. Escritos sobre la biología y su enseñanza, 10 (19), 21-40.

Fonteyn, M., Kuipers, B. y Grobe, S. (1993). A description of think-aloud method and protocol analysis. Qualitative Health Research, 3 (4), 430-441. 
Gess-Newsome, J. y Lederman, D. (1999). Examining Pedagogical Content Knowledge. The construct and its implications for science education. Dordrecht, The Netherlands: Kluwer Academic Press.

Grossman, P. (2005). Un estudio comparado: las fuentes del Conocimiento Didáctico del Contenido en la enseñanza del inglés. Profesorado. Revista de Currículum y Formación del profesorado, 9 (2), 1-18.

Guerrero, J., Castillo, E., Chamorro, H. e Isaza, G. (2013). El error como oportunidad de aprendizaje desde la diversidad en las prácticas evaluativas. Plumilla educativa, 12 (2), 361-381.

Gumperz, J. (1982). Discourse Strategies. Oxford, UK: Oxford University Press.

Hymes, D. (1962). The ethnography of speaking. En T. Gladwin y W. Sturtevant (Eds.), Anthropology and Education (pp. 15-53). Washington: Anthropological Society of Washington.

Kawulich, B. (2006). La observación participante como método de recolección de datos. Forum: Qualitative Social Research, 6 (2), Art. 43. Recuperado de www. qualitative-research.net/index.php/fqs/article/ download/466/999

Lave, J. y Wenger, E. (1991). Situated learning. Legitimate peripheral participation. New York: Cambridge University Press. Traducido por: Alfaro, C. [Internet] Recuperado de http://www.universidad-de-la-calle. com/Wenger.pdf

Medina, J. L. y Jarauta, B. (2013). Análisis del Conocimiento Didáctico del Contenido de tres profesores universitarios. Revista de Educación, 360, 600-623. [Internet] Recuperado de http://www.mecd.gob.es/dctm/ revista-de-educacion/articulosre360/re36027. pdf?documentId=0901e72b814a77fb

Medina, J. (2014). El proceso de comprensión en el análisis de datos cualitativos en educación. Magis. Revista Internacional de Investigación en Educación, 7(14), 39-54.

Nitti, M., Ciavolino, E., Salvatore, S. y Gennaro, A. (2010). Analyzing psychotherapy process as intersubjective sensemaking: an approach based on discourse analysis and neural networks. Psychotherapy Research: Journal of the Society for Psychotherapy Research, 20 (5), 546-563. doi:10.1080/10503301003641886

Santibáñez, C. (2015). Robustez como categoría para el análisis de la cognición: el caso de la competencia argumentativa. Cinta Moebio, 52, 60-68 www.moebio. uchile.cl/52/santibanez.html
Shulman, L. (1987). Knowledge and teaching: foundations of the New Reform. Harvard Educational Review, 57 (1), $1-22$.

Shulman, L. (2004). Teaching as community property. Essays on Higher Education. San Francisco: Jossey Bass.

Schön, D. A. (1992). La formación de profesionales reflexivos. Hacia un nuevo diseño de la formación y el aprendizaje en las profesiones. Madrid: Paidós Ibérica.

Schön, D. A. (1998). El profesional reflexivo: cómo piensan los profesionales cuando actúan. Barcelona. Paidós Ibérica.

Schubert, V., Menegaz, J., Carvalho, F., Costa, L., Pareto, A. y Souza, S. (2017). LeeShulman: Contribuições para a investigação da formação docente em enfermagem e saúde. Texto \& Contexto - Enfermagem, 26(4), e1080017.https:// dx.doi.org/10.1590/0104-07072017001080017

Smith, D.y Neale, C. (1989). The construction of subject matter knowledge in primary science teaching. Teaching and Teacher Education, 5 (1), 1-20.

Taylor, S. J. y Bogdan, R. (1987). Introducción a los métodos cualitativos de investigación. Barcelona: Paidós.

Valles, M. (1999). Técnicas cualitativas de investigación social. Madrid: Síntesis.

van Zee, E. y Minstrell, J. (1997a). Reflective discourse: developing shared understandings in a physics classroom. International Journal of Science Education, 19 (2), 209-228.

van Zee, E. y Minstrell, J. (1997b). Using questioning to guide student thinking. Journal of the Learning Sciences, 6 (2), 227-269.

Vygotsky, L. (2010). Pensamiento y lenguaje. Barcelona: Paidós.

Wassermann, S. (1994). Introduction to case method teaching. Nueva York: Teachers College Press, Columbia University.

Weston, C., y McAlpine, L. (2002). Parallel transcripts: an innovative approach for capturing the impact of reflective teaching on student learning experience. En Annual Meeting of the American Education Research Association. New Orleans.

Woods, P. (1987). La escuela por dentro: La etnografía en la investigación educativa. Barcelona: Paidós Ibérica.

Yang, M.y Carless, D. (2013). The feedback triangle and the enhancement of dialogic feedback processes. Teaching in Higher Education, 18 (3), 285-297. 